Article

\title{
Understanding the Energy Consumption of Battery Electric Buses in Urban Public Transport Systems
}

\author{
Shefang Wang ${ }^{1}{ }^{\oplus}$, Chaoru Lu ${ }^{2, *}$, Chenhui Liu ${ }^{3}$, Yue Zhou ${ }^{4}$, Jun Bi ${ }^{5}$ and Xiaomei Zhao ${ }^{5}$ \\ 1 BASF Corporation, Iselin, NJ 08830, USA; shefang.wang@basf.com \\ 2 Department of Civil Engineering and Energy Technology, Oslo Metropolitan University, 0167 Oslo, Norway \\ 3 Turner-Fairbank Highway Research Center, McLean, Fairfax County, VA 22101, USA; \\ chenhui.liu.ctr@dot.gov \\ 4 C2SMART Center, New York University, Brooklyn, NY 11201, USA; yz2065@nyu.edu \\ 5 School of Traffic and Transportation, Beijing Jiaotong University, Beijing 100044, China; jbi@bjtu.edu.cn (J.B.); \\ xmzhao@bjtu.edu.cn (X.Z.) \\ * Correspondence: chaorulu@oslomet.no; Tel.: +47-40-18-71-92
}

Received: 14 October 2020; Accepted: 27 November 2020; Published: 30 November 2020

\begin{abstract}
The ever-increasing concerns over urban air quality, noise pollution, and considerable savings in total cost of ownership encouraged more and more cities to introduce battery electric buses (e-bus). Based on the sensor records of 99 e-buses that included over 250,000 $\mathrm{h}$ across 4.7 million kilometers, this paper unveiled the relationship between driving behaviors and e-bus battery energy consumption under various environments. Battery efficiency was evaluated by the distance traveled per unit battery energy ( $1 \%$ SoC, State of Charge). Mix effect regression was applied to quantify the magnitude and correlation between multiple factors; and 13 machine learning methods were adopted for enhanced prediction and optimization. Although regenerative braking could make a positive contribution to e-bus battery energy recovery, unstable driving styles with greater speed variation or acceleration would consume more energy, hence reduce the battery efficiency. The timing window is another significant factor and the result showed higher efficiency at night, over weekends, or during cooler seasons. Assuming a normal driving behavior, this paper investigated the most economical driving speed in order to maximize battery efficiency. An average of $19 \%$ improvement could be achieved, and the optimal driving speed is time-dependent, ranging from 11 to $18 \mathrm{~km} / \mathrm{h}$.
\end{abstract}

Keywords: electric bus; battery efficiency; driving behavior; mixed effect regression; machine learning methods

\section{Introduction}

Compared to conventional diesel or compressed natural gas buses, one of the biggest challenges nowadays for e-buses is their high upfront cost $(\$ 570,000$ vs. $\$ 450,000)$; however the cost parity was forecasted to be reached before 2030 [1]. Total cost of ownership (TCO) of an e-bus includes the upfront cost and additional operating cost over the lifespan. Many factors affect the TCO, including electricity prices, average driving distances, charging configuration, and financing costs. The research discovered that longer traveling distances favor e-bus and as battery prices keep on falling, the e-bus will become more and more competitive on a TCO basis [1,2]. On top of the competitive TCO, e-buses also have health and environmental benefits, because they do not produce tailpipe pollution and make much less noise [3,4]. Due to these benefits, many European countries are accelerating the transformation of zero emission vehicles (ZEV). For example, by 2025, Norway is aiming for transitioning to all ZEVs. Following Norway, six other counties are targeting to achieve the transformation in 2030, including Denmark, Iceland, Ireland, Netherlands, Slovenia, and Sweden [5]. The European Commission and the 
Committee of the Regions launched a dedicated initiative for clean buses-the Clean Bus Deployment Initiative. Since then, many cities and regions have announced plans to stop purchasing fueled buses, like Copenhagen, London, Berlin, and Oslo [6].

Although electric vehicles (EV) are environmental-friendly and able to benefit the development of a sustainable urban transit system, an important concern is energy consumption estimation that related to driver's range anxiety-avoid driving with little energy remaining in the battery [7-9]. Energy consumption can be quantified via battery state of charge $(\mathrm{SoC})$, which reflects the level of charge compared to its capacity. SoC is scaled from $100 \%$ to $0 \%$, where $100 \%$ represents a fully charged status and $0 \%$ refers to an empty battery with no energy. EV energy consumption is driven by multiple aspects. For example, the average travel speed and acceleration were rated by researchers [10] and they found that the energy consumption for a passenger EV was extremely high when the average speed was below $20 \mathrm{~km} / \mathrm{h}$. Another case study in Beijing, China [9], analyzed the driving distance per SoC (DDPSoC) using a four-door passenger EV. They applied robust nonlinear regressions to fit speed and DDPSoC under three temperature conditions and identified $50 \mathrm{~km} / \mathrm{h}$ as the most economical driving speed that can reach the maximum remaining driving range. Both of the above-mentioned papers advised higher speed to improve battery efficiency; but such speed strategies were designed for passenger EVs. As a subset of EVs, e-buses mainly focus on moving people along stations on predetermined routes and frequent stops are needed to serve passengers; therefore, this study hypothesized that e-bus optimal speed strategy might be different from passenger EV. Research found that more than $30 \%$ of the energy could be consumed by air conditioning, so the operational range of e-bus is significantly influenced by the use of air conditioners [11,12]. Furthermore, the bus operator's driving behavior is another critical component, because abrupt acceleration or deceleration consumes more battery power, which can greatly decrease the operational range [12,13].

After identifying potential factors (speed, temperature, air conditioning, and acceleration styles) that linked to e-bus battery energy consumption from prior literature, statistical models can help to understand the correlation. Different buses were operating in numerous routes under a variety of roadway traffic conditions; however, detailed information was difficult to collect and measure directly. For example, detailed information like roadway geometry along the bus route, detailed hourly city weather conditions, passengers carried, etc., are not available for the current study. To incorporate the vehicle-specific correlations and unobserved driving heterogeneity, a mixed effect modeling framework was employed to understand the relationship between various factors and the target variable. The modeling results are highly interpretable and widely used in many naturalistic driving studies [14-17] and EV researches, like charging behavior studies [18-20] and energy consumption estimation [21].

Besides the mixed effect models, machine learning algorithms were also frequently utilized in many EV research areas because of the enhanced prediction accuracy; however, the model's interpretability is compromised [22]. For instance, both support vector machine (SVM) and multi-layer neural network were applied to assess battery aging for real-world e-buses [23]. To predict charging demand at EV charging stations, Decision Tree and Random Forest regressors were adopted [24,25]. Random Forest regression can also estimate lithium-ion battery state-of-health [26] and state-of-charge [27]. Apart from Random Forest, other ensemble methods like Gradient Boosting Tree were used for battery remaining useful life prediction [28] and XGBoost algorithm for battery SoC estimation [29]. These ensemble methods (e.g., XGBoost and Random Forest) were showing better accuracy for the research on EV energy consumption, compared with linear family models (Lasso, Ridge, and Elastic Net) [30].

While prior battery energy consumption researches investigated multiple factors and analysis methods, they were often focusing on the passenger EVs and limited by the sample size. The present paper will extend existing research efforts on e-buses by evaluating a rich driving dataset collected from 99 e-buses in seven cities during a long-term (15 months) period. Due to the unobserved driving heterogeneity, the linear mixed effect regression was adopted in the result section to understand hidden insights that are crucial to battery efficiency of e-buses. Studies proposed optimal driving speed 
strategies for passenger EVs; however, the present study hypothesized that such speed might not be suitable for e-bus operations in urban public transport systems. After training and identifying the most predictable machine learning model, an optimized bus traveling speed strategy will be presented in the following section.

\section{Materials and Methods}

More than one year (from May 2017 to July 2018) of operational data were collected from 99 e-buses that were running at seven different cities in China. All of them were the same type (Table 1) and the in-vehicle sensors, GPS (Global Positioning System) and BMS (Battery Management System), were recording bus status every $10 \mathrm{~s}$. For the average historical temperature (daily high, low, and average), Figure 1 illustrates the distribution of seven cities in this study.

Table 1. E-bus specifications.

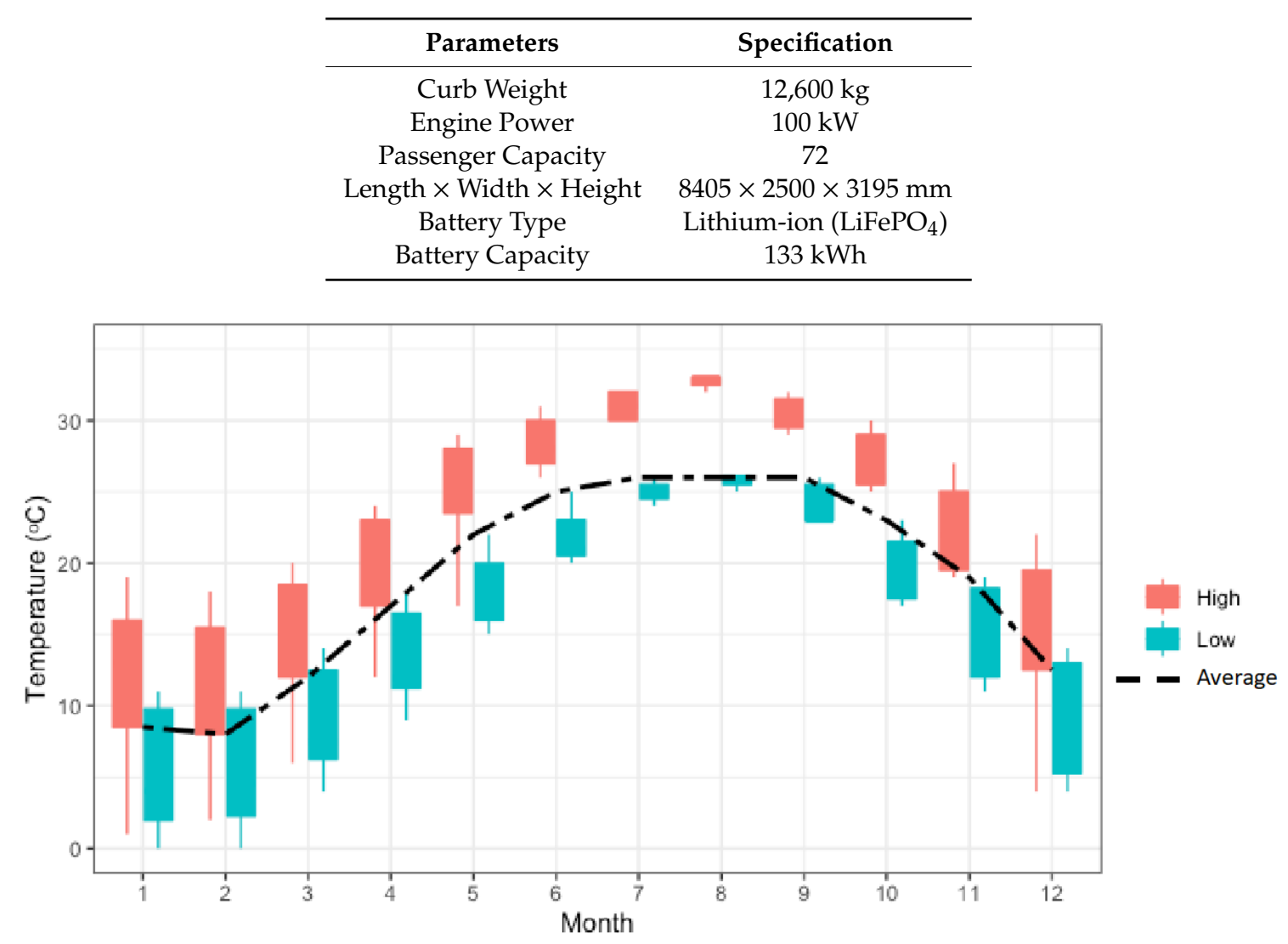

Figure 1. Average historical daily temperature-high, low, and average.

This paper aims to analyze battery performance when a bus is running along the road; therefore, data were aggregated based on the battery discharge period-it started as a bus left the charging station or restarted, covered the driving time in the city transit network, and ended as a bus was switched off. Figure 2 visualizes example driving profiles in two afternoons between 2 p.m. and 4 p.m.; and in each afternoon, this particular bus experienced two battery discharge periods and one charging status in-between. For each discharge period, several features were calculated, including average speed, standard deviations of acceleration and deceleration, mileage, and SoC (State of Charge). Using Figure 2 as an example, Table 2 summarizes statistics for each battery discharge period and the calculated distance per SoC will be treated as the target variable in the following section. 

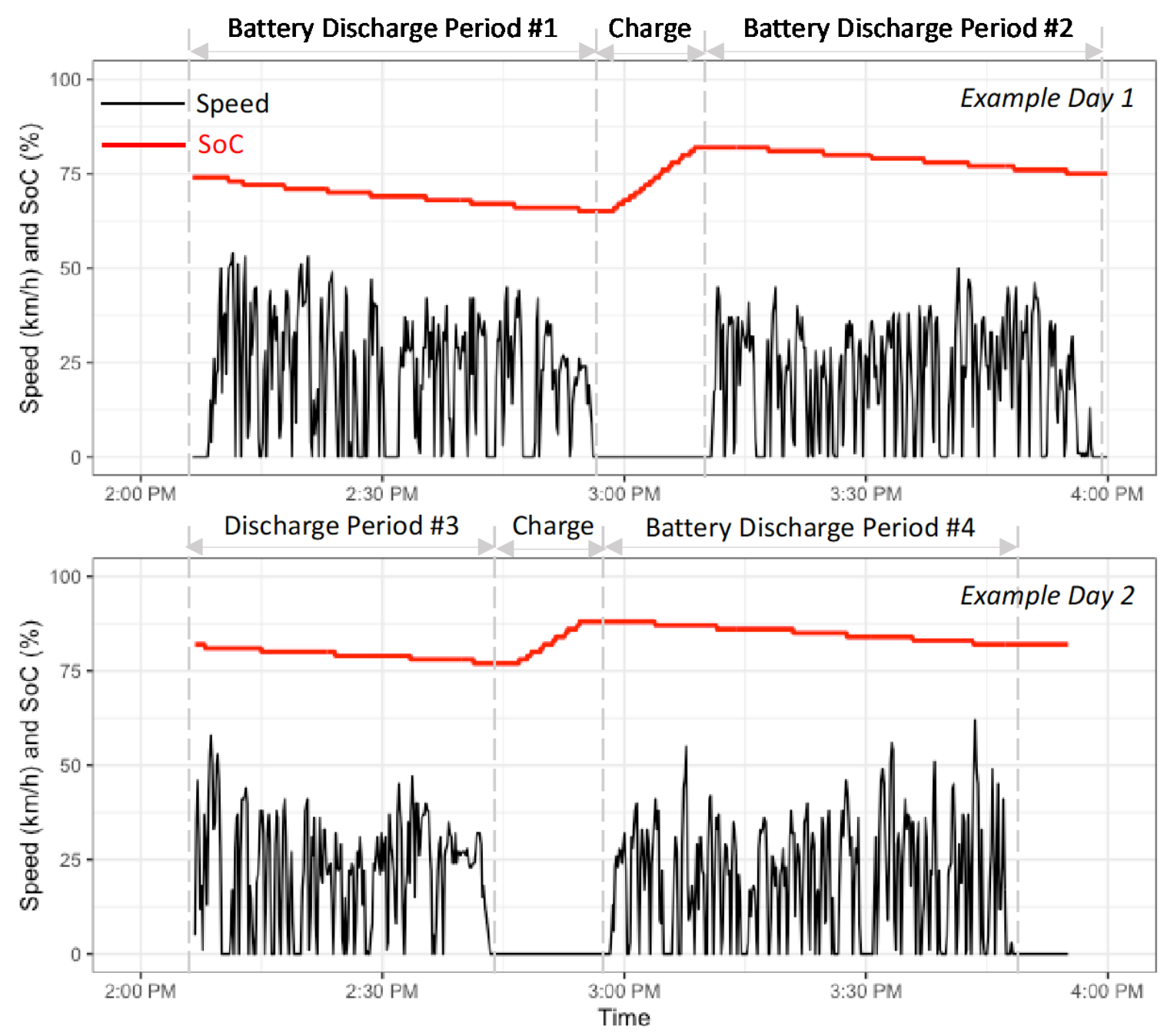

Figure 2. Example driving profile.

Table 2. Example summary statistics for four battery discharge periods from Figure 2.

\begin{tabular}{ccccc}
\hline Features & Period \# & Period \# 2 & Period \# 3 & Period \# 4 \\
\hline SoC Start (\%) & 74 & 82 & 82 & 88 \\
SoC End (\%) & 65 & 75 & 77 & 82 \\
Mileage Start (km) & 5044 & 5062 & 7152 & 7166 \\
Mileage End (km) & 5062 & 5079 & 7166 & 7182 \\
Average Speed (km/h) & 20.82 & 20.03 & 21.98 & 19.92 \\
Speed Standard Deviation (Std) & 16.09 & 14.63 & 14.39 & 15.31 \\
Overall Acceleration Std. & 0.39 & 0.35 & 0.37 & 0.41 \\
Deceleration Std. & 0.28 & 0.25 & 0.26 & 0.29 \\
Distance per SoC (km) & $(5062-5044) /$ & $(5079-5062) /$ & $(7166-7152) /$ & $(7182-7166) /$ \\
& $(74-65)=2.00$ & $(82-75)=2.64$ & $(82-77)=2.80$ & $(88-82)=2.67$ \\
\hline
\end{tabular}

After data cleaning and aggregation, a total of 62,057 battery discharge periods were extracted, covering 4,727,206 km traveled over $256,979 \mathrm{~h}$. Table 3 provides the summary statistics of all continuous modeling variables and three time-related categorical covariates that were extracted from GPS time stamps. One is for meteorological temperate seasons, where Spring covers from March to May, with a historical average daily temperature from 12 to $22{ }^{\circ} \mathrm{C}$; Summer $\left(25-26{ }^{\circ} \mathrm{C}\right)$ ranges from June to August, Fall $\left(19-26^{\circ} \mathrm{C}\right)$ is between September and November, and Winter $\left(8-12^{\circ} \mathrm{C}\right)$ lasts from December till February. The other is the time of day, where the daytime starts at 6 a.m. and the nighttime begins at 6 p.m. The third one considers weekdays (Monday to Friday) and weekends (Saturday or Sunday). 
Table 3. Summary statistics of modeling variables.

\begin{tabular}{|c|c|c|c|c|c|}
\hline Variables & Mean & Std. & Range & Count & Description \\
\hline Distance per SoC $(\mathrm{km})$ & 2.19 & 0.41 & $1.38-3.40$ & - & Target variable \\
\hline Average Speed (km/h) & 19.88 & 3.42 & $5.66-42.21$ & - & \multirow{4}{*}{$\begin{array}{l}\text { Factors that reflect } \\
\text { driving behaviors }\end{array}$} \\
\hline $\begin{array}{l}\text { Speed Standard } \\
\text { Deviation (Std.) }\end{array}$ & 18.75 & 2.34 & $10.39-31.03$ & - & \\
\hline Overall Acce. Std. & 0.41 & 0.10 & $0.12-1.46$ & - & \\
\hline Deceleration Std. & 0.38 & 0.15 & $0.12-2.61$ & - & \\
\hline SoC Start (\%) & 89.07 & 12.36 & $14-100$ & - & \multirow{2}{*}{ Battery state of charge } \\
\hline SoC End (\%) & 53.76 & 18.30 & $11-97$ & - & \\
\hline Spring $\left(12-22^{\circ} \mathrm{C}\right)$ & - & - & - & 17,656 & \multirow{4}{*}{$\begin{array}{c}\text { Categorical seasonal } \\
\text { factors }\end{array}$} \\
\hline Summer $\left(25-26^{\circ} \mathrm{C}\right)$ & - & - & - & 19,338 & \\
\hline Fall $\left(19-26^{\circ} \mathrm{C}\right)$ & - & - & - & 14,354 & \\
\hline Winter $\left(8-12{ }^{\circ} \mathrm{C}\right)$ & - & - & - & 10,709 & \\
\hline Daytime & - & - & - & 59,810 & \multirow{2}{*}{ Time of day } \\
\hline Nighttime & - & - & - & 2247 & \\
\hline Weekday & & & & 44,058 & Weekday (Mon. to Fri.) \\
\hline Weekend & & & & 17,999 & Weekend (Sat. \& Sun.) \\
\hline
\end{tabular}

The general form of linear mixed effect model in matrix notation is listed in the following equation [31]:

$$
y=X \beta+Z u+\varepsilon
$$

where $y$ is the target variable with size $N \times 1 ; X$ is a $N \times p$ matrix with $p$ predictors; $\beta$ is the fixed-effect coefficients with size $p \times 1 ; Z$ is the random complement to the fixed $X$ with size $N \times q$ and $u$ is a $q \times 1$ column vector of the random effects. $\varepsilon$ is the residuals vector that accounts for the randomness that cannot be explained by $\mathbf{X} \boldsymbol{\beta}+\mathbf{Z u}$. In this study, the bus-specific correlations need to be considered to accommodate unobserved driving heterogeneity. The final modeling dataset has 62,057 records $(N=62,057)$ with 99 e-buses $(q=99)$. All the continuous input variables were z-scored to allow effect comparison across a uniform scale and the R package lme4 (Linear Mixed Effects Models using 'Eigen' and S4) [32] was used to fit the model.

\section{Results}

In this session, the linear mixed effect regression model was estimated to quantify the magnitude and correlation between multiple factors and battery efficiency. Then, after hyper-parameter fine-tuning, training, random sampling, and comparing 13 different machine learning algorithms, the Random Forest was identified as the most predictable method. At last, the Random Forest regressor was further adopted to search the optimal speed using the stochastic differential evolution optimization.

\subsection{Understanding Correlations Using Mixed Effect Modeling}

Table 4 presents mixed effect modeling results for average distance traveled per SoC. The average speed is statistically significant, and its positive coefficient indicates that the faster the speed, the longer distance can be traveled per unit battery energy. The standard deviation of bus operation speed and acceleration can reflect operator's driving behaviors-the higher the speed variation, the greater the driving fluctuations, and the higher the acceleration, the more excessive sudden pressing on brake or gas pedals. Both of them have significant negative effects, suggesting that unstable driving behavior can greatly increase battery energy consumption; hence reduce the distance traveled. The seasonal indicator was treated as a categorical variable and the baseline is Spring. All of them are significant, showing that environmental temperature plays an important role in battery energy consumption. It is possible to assume that the higher the temperature (in Summer), the heavier usage of bus air 
conditioners, thus increasing the energy consumptions and shortening the total distance that can be reached. More people use buses during daytime or weekdays, hence battery energy could be exhausted faster with the heavier load. The positive coefficient of nighttime further validates such a hypothesis-with carrying fewer passengers at night or weekends, the bus can travel longer.

Table 4. Mixed effect modeling results.

\begin{tabular}{ccccc}
\hline & Estimate & Std. Error & t Value & Prob. $(>|\mathbf{t}|)$ \\
\hline Intercept & 2.273 & 0.025 & 90.02 & $<0.001$ \\
Speed Average & 0.047 & 0.002 & 25.43 & $<0.001$ \\
Speed Std. & -0.020 & 0.003 & -7.86 & $<0.001$ \\
Overall Acceleration Std. & -0.057 & 0.002 & -26.48 & $<0.001$ \\
Deceleration Std. & 0.041 & 0.002 & 20.42 & $<0.001$ \\
SoC Start & 0.027 & 0.002 & 16.62 & $<0.001$ \\
SoC End & 0.050 & 0.002 & 28.47 & $<0.001$ \\
Spring $\left(12-22{ }^{\circ} \mathrm{C}\right)$ & Baseline & & & \\
Summer $\left(25-26^{\circ} \mathrm{C}\right)$ & -0.289 & 0.0031 & -88.67 & $<0.001$ \\
Fall $\left(19-26{ }^{\circ} \mathrm{C}\right)$ & -0.057 & 0.0034 & -16.21 & $<0.001$ \\
Winter $\left(8-12{ }^{\circ} \mathrm{C}\right)$ & 0.086 & 0.0037 & 22.88 & $<0.001$ \\
Daytime $(6$ am-6 pm) & Baseline & & & \\
Nighttime $(6$ pm-6 am) & 0.143 & 0.0072 & 19.42 & $<0.001$ \\
Weekend (Sat. or Sun.) & Baseline & & & \\
Weekday (Mon. to Fri.) & -0.007 & 0.0026 & -2.43 & 0.015 \\
\hline
\end{tabular}

\subsection{Predicting Energy Consumption via Machine Learning}

While mixed effect regression model is great for factor interpretation, the prediction accuracy might be compromised due to its linear nature. In this section, several machine learning algorithms (including both linear and non-linear methods) are assessed to explore the model's prediction accuracy.

Figure 3 illustrates the framework used in the current paper for model hyperparameter tuning, mode selection, and model validation. Thirteen machine learning estimators were tested, including linear regressors (Linear Regression, Lasso, Ridge, Elastic Net, and Robust Huber Regression), kernel-based method (Support Vector Machine), method with dimension reduction (PLS), multi-layer perceptron (MLP) and deep neural network with dropout, tree-based method (Decision Tree), and ensemble methods (Random Forest, Boosting Trees, and XGBoost). It is desired to identify the most predictive method for the unseen data; therefore, the whole dataset was randomly divided into training, validation, and testing samples, based on the ratio of $60 \%, 20 \%$, and $20 \%$. Each estimator has a set of hyper-parameters (the parameter that used to control the learning process) and they are tuned via an exhaustive grid search based on the training and validation dataset. Additionally, to consider the randomness, this process was repeated 50 times via random sampling. Within each iteration, $80 \%$ of the data were used to fine-tune the model and the remaining unseen $20 \%$ were reserved as the testing data to verify the model's performance.

Mean Square Error (MSE), the average squared difference between the predicted values and the actual values, was chosen as modeling performance in Figure 4. The Random Forest regressor outperforms other machine learning methods as evidenced by the lowest MSE scores for both training and testing dataset. Figure 4 also embeds two parity plots for Random Forest at one iteration during the process of random sampling, where the $x$-axis represents true values and $y$-axis is prediction. The bottom one is for training performance and upper plot is based on testing dataset. Overall, the model performance is good, as all the points clustered along the $45^{\circ}$ diagonal line. However, some systematic bias was observed, as the regressor overestimates (prediction is higher than true value) at the lower end and underestimates (prediction is lower than true value) at the higher end. 


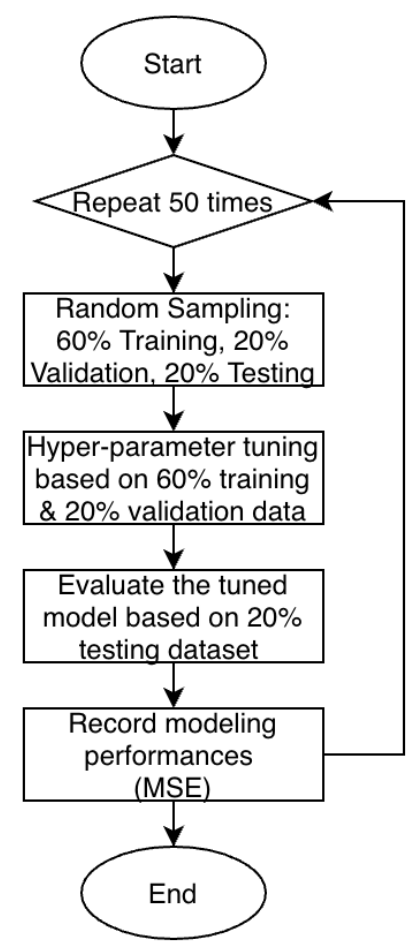

Figure 3. Framework for model hyperparameter tuning, mode selection, and model validation.
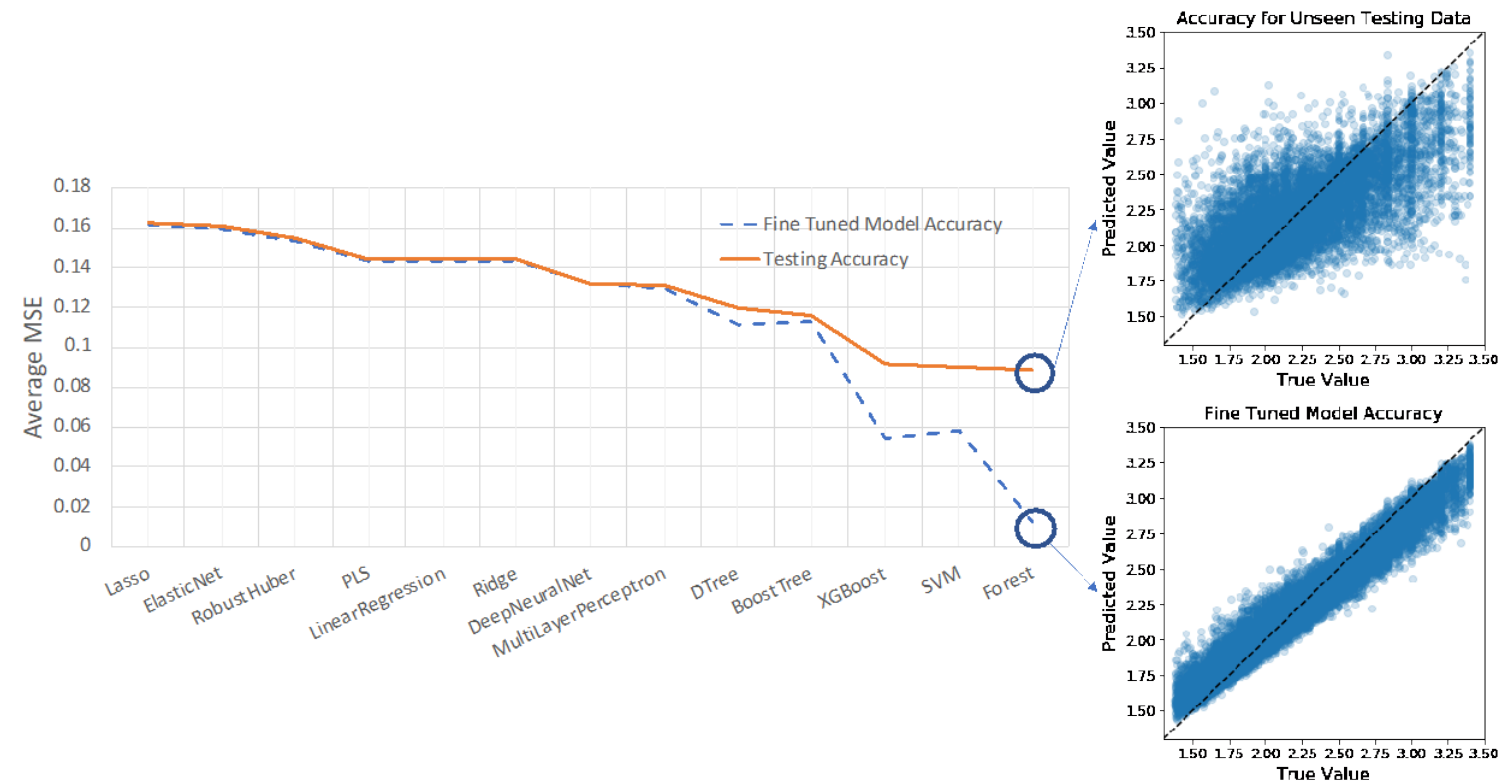

Figure 4. Average MSE (Mean Square Error) for 13 different regression methods.

\subsection{Optimization to Identify the Most Economical Driving Speed}

Once the random forest estimator was fine-tuned and selected, the next question is to optimize the target variable-maximizing total distance traveled per SoC (DPSoC) to achieve a higher battery efficiency. Although there are multiple different input features for the random forest regressor, this study aims to find the most economical driving speed based on typical driving behavior and driving environment during weekdays. The median values were adopted to represent a typical driving behavior - an operator started a bus with 97\% charge, after driving it normally (with $18.64 \mathrm{~km} / \mathrm{h}$ as speed standard deviation, 0.39 as overall acceleration variation, and 0.33 as deceleration variation) along city transit network, this bus consumed $43 \%$ battery energy and returned to charging station 
with an ending SoC of $54 \%$. As discovered by the statistical model, timing is critical to e-bus better performance; therefore, the optimization was conducted based on eight different situations:

$$
\begin{aligned}
& \underset{\text { speed }_{\text {max }}}{\operatorname{maPSoC}}
\end{aligned}
$$

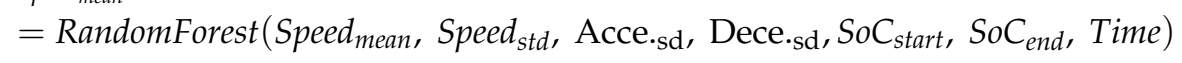

$$
\begin{aligned}
& \text { subject to }: \text { Speed }_{\text {sd }}=18.64, \text { Acce }_{\text {sd }}=0.39, \text { Dece }_{\text {sd }}=0.33, \text { SoC }_{\text {start }}=97 \%, \text { SoC }_{\text {end }} \\
& =54 \% \text {, Time }
\end{aligned}
$$

Equation (2) summarizes objective function which subjects to some normal driving assumptions. Random Forest is an ensemble black-box model with limited information of gradient. Differential evolution optimization was utilized because it is a stochastic method and does not depend on the gradient to find the optimum [33]. Table 5 provides the optimization results with respect to different time windows. It was impossible to find the identical constrains (speed variation, acceleration/deceleration, and SoC as listed in Equation (2) from the historical dataset; therefore, the existing unoptimized distance per SoC was approximated by averaging all the data points around the constrains within a $\pm 2.5 \%$ buffer (increase the buffer by a step of $0.25 \%$ if no data point was identified). An average of $19.5 \%$ improvement was observed after applying the most economical speed strategy.

Table 5. The most economical e-bus speed.

\begin{tabular}{cccccc}
\hline Season & Time & $\begin{array}{c}\text { Most Economical } \\
\text { Speed } \mathbf{( k m} / \mathbf{h})\end{array}$ & $\begin{array}{c}\text { Maximum Distance } \\
\text { per SoC } \mathbf{( k m )}\end{array}$ & $\begin{array}{c}\text { Approximated Existing } \\
\text { Distance per SoC (km) }\end{array}$ & $\begin{array}{c}\text { Improve } \\
\mathbf{( \% )}\end{array}$ \\
\hline Spring & Day & 11.13 & 2.35 & 2.19 & $7.4 \%$ \\
Spring & Night & 11.11 & 2.36 & 1.65 & $42.8 \%$ \\
Summer & Day & 18.48 & 1.95 & 1.89 & $3.5 \%$ \\
Summer & Night & 32.15 & 1.96 & 2.21 & $-11.1 \%$ \\
Fall & Day & 17.30 & 2.31 & 1.89 & $22.1 \%$ \\
Fall & Night & 17.45 & 2.30 & 2.34 & $-1.4 \%$ \\
Winter & Day & 11.49 & 2.36 & 1.56 & $51.1 \%$ \\
Winter & Night & 11.39 & 2.36 & 1.67 & $41.3 \%$ \\
\hline
\end{tabular}

\section{Discussion}

There were approximately 385,000 e-buses globally in 2017 and 99\% of them were operated in China [1]. Inspired by 99 e-buses from seven cities in China, the present paper treated the total distance traveled per unit $\mathrm{SoC}$ as a surrogate for battery efficiency and researched its correlations with driving behaviors under various environments.

Statistical model with mixed effects can consider the unobserved driving heterogeneity and quantify the correlation and magnitude of each factor. Specifically, the average driving speed is essential to battery efficiency, and the higher the speed, the longer distance could be traveled. Both speed variation and acceleration can reflect driving behavior, which could be attributable to operator's intrinsic driving style or traffic conditions. Their modeling coefficients were negative, indicating that the more unstable maneuvers that were performed, the shorter distance can be covered per unit battery energy. Dedicated bus lanes (DBL) can free buses from traffic interference [34] and the study found that it could save bus running time and reduce the operation variability [35]. To provide a better driving environment and enhance battery efficiency further, DBL is recommended, because operators can drive faster and smoother. As for the phenomenon of regenerative braking, this should be partially reflected by the standard deviation of deceleration during each battery discharge period. Its coefficient is positive, suggesting that regenerative braking can make a positive contribution to e-bus battery energy recovery. 
In addition to different driving behaviors, this paper also identified strong correlations between battery energy consumption and timing windows. Temporal factors like time of day and day of week are considerable, and e-buses can travel $6.3 \%$ longer per unit battery energy at night and $0.3 \%$ longer over weekends. This improvement makes sense because the daytime traffic is always more congested than nighttime and with fewer passengers on the bus, the battery can last longer. Seasonal effect is another dominant factor. Assuming Spring is the baseline, the battery efficiencies were reduced during hotter months- $12.7 \%$ and $2.5 \%$ reduction for Summer and Fall, respectively, whereas battery efficiency was increased by $3.8 \%$ during Winter. It is well acknowledged that a lithium-ion battery cell often performs worse at a lower temperature due to the increased resistance. However, most of the buses in the current study were operated in warm southern cities in China, where the average temperature during Winter is around $10^{\circ} \mathrm{C}$. Although the detailed data is not available, it is highly possible that the major battery efficiency reduction during Summer is caused by air conditioning usage.

While mixed effect model helps to understand correlations among existing historical data, its ability to predict the unknown is limited. After a comprehensive evaluation of 13 machine learning algorithms, the Random Forest was chosen because of its lowest modeling errors in both training (understanding existing patterns) and testing (predicting unknown) datasets. It was integrated into the next analysis stage-identifying the optimum speed that can assist bus drivers to travel longer within a unit SoC. Results found that the average maximum distance which could be achieved is about $2.24 \mathrm{~km}$ per unit battery energy, with a reduction in Summer (1.96) and increase in Spring (2.36), Fall (2.31) and Winter (2.36). As hypothesized, the optimal speed strategy for e-bus is much lower than the previous researches on passenger EVs; and an average of $19.5 \%$ improvement could be achieved based on the most economical speed under a normal driving style. Unlike passenger EVs, e-bus serves as public transportation and our results can provide a scientific guidance to help bus drivers better control e-bus with a greater energy efficiency.

\section{Conclusions}

This paper processed a comprehensive dataset (55 GB) that is composed of the data collected from 99 e-buses in seven cities in China over one year. Various driving behaviors (average speed, speed variation, and acceleration) were linked to battery efficiency and a stable driving style would be appreciated for a higher efficiency. Different timing windows were also proven to achieve a higher battery efficiency, probably due to fewer passengers at night and less air conditioning usage during cooler seasons.

By analyzing historical datasets, one can learn from prior experiences; but more importantly, one wants to forecast the unknown future. This paper introduced a framework that starts with understanding existing data via mixed effect regression; followed by a comprehensive model selection and evaluation, in order to pick up a method with the most prediction power. This case study pinpointed the Random Forest to be the best candidate and applied it in the optimization stage for a more productive battery usage plan. The findings of the most economical speed were recommended, which could be served as an actionable bus operating guideline during different times.

As the current paper only analyzed a portion of dataset, more actions are planned in the future; for example, including the battery details (current and voltage information) and engine status (rotation speed and torque). Neural network methods (MLP and deep neural net) were tested in the current study; however, their performances were not good enough. With a much more complex layer structure (activation function, dropout rates, learning rates, etc.) to explore, it is possible that the current research did not identify the suitable deep learning models. More deep learning methods will be studied by the team in the future. GPS data were collected but the GIS (Geographic Information System) roadway profile data was not available for the current study, which made it impossible to compare battery performance under various roadway conditions (ramp, straight/curved road, or slope). Although mixed effect model can account for these unobserved driving heterogeneities to some extent, further study is recommended based on the roadway profile data. Admittedly, temperature has a 
great influence on the battery energy consumption; however, the current research only considered the seasonal aspect, which lost the detailed insights in daily or hourly levels. Although it is a lengthy process, the research team is currently collecting detailed weather information (wind speed, temperature, precipitation, etc.) from each city in a much granular level to further extend the research in the future.

Author Contributions: S.W.: Conceptualization, Methodology, Data curation, Formal analysis, Writing-original draft. C.L. (Chaoru Lu): Conceptualization, Funding acquisition, Methodology, Writing-review \& editing. C.L. (Chenhui Liu): Conceptualization, Data curation, Writing-review \& editing. Y.Z.: Conceptualization, Data curation, Writing-review \& editing. J.B.: Conceptualization, Funding acquisition, Data curation, Writing-review \& editing. X.Z.: Conceptualization, Funding acquisition, Data curation, Writing-review \& editing. All authors have read and agree to the published version of the manuscript.

Funding: The extensive data collection was supported by the JPI Urban Europe-NSFC project named SMUrTS. The project was funded by the National Natural Science Foundation of China (grant number 71961137008) and the Research Council of Norway (grant number 299078).

Conflicts of Interest: The authors declare no conflict of interest.

\section{References}

1. Bloomberg New Energy Finance. Electric Buses in Cities: Driving Towards Cleaner Air and $\mathrm{Lower}^{\mathrm{CO}}$. 2018. Available online: https://assets.bbhub.io/professional/sites/24/2018/05/Electric-Buses-in-Cities-ReportBNEF-C40-Citi.pdf (accessed on 28 November 2020).

2. Meishner, F.; Sauer, D.U. Technical and economic comparison of different electric bus concepts based on actual demonstrations in European cities. IET Electr. Syst. Transp. 2020, 10, 144-153. [CrossRef]

3. Kühne, R. Electric buses-An energy efficient urban transportation means. Energy 2010, 35, 4510-4513. [CrossRef]

4. Environmental and Energy Study Institute. Battery Electric Buses: Benefits Outweigh Costs. 2018. Available online: https://www.eesi.org/files/FactSheet_Electric_Bus_Benefits_Outweigh_Costs_1018.pdf (accessed on 14 November 2020).

5. Slowik, P.; Hall, D.; Lutsey, N.; Nicholas, M.; Wappelhorst, S. Funding the Transition to All Zero-emission Vehicles. 2019. Available online: https://theicct.org/sites/default/files/publications/Funding_transition_ZEV_ 20191014.pdf (accessed on 14 November 2020).

6. EU. European Clean Bus Deployment Initiative. 2020. Available online: https://ec.europa.eu/transport/ themes/urban/cleanbus_en (accessed on 14 November 2020).

7. Pearre, N.S.; Kempton, W.; Guensler, R.L.; Elango, V.V. Electric vehicles: How much range is required for a day's driving? Transp. Res. Part C Emerg. Technol. 2011, 19, 1171-1184. [CrossRef]

8. Liu, K.; Yamamoto, T.; Morikawa, T. Impact of road gradient on energy consumption of electric vehicles. Transp. Res. Part D Transp. Environ. 2017, 54, 74-81. [CrossRef]

9. Bi, J.; Wang, Y.; Sai, Q.; Ding, C. Estimating remaining driving range of battery electric vehicles based on real-world data: A case study of Beijing, China. Energy 2019, 169, 833-843. [CrossRef]

10. Zhang, R.; Yao, E. Mesoscopic model framework for estimating electric vehicles' energy consumption. Sustain. Cities Soc. 2019, 47, 101478. [CrossRef]

11. United Nations Environment Programme. UNEP Environmental Assessment Expo 2010 Shanghai, China. 2010. Available online: https://stg-wedocs.unep.org/handle/20.500.11822/7777?show=full (accessed on 14 November 2020).

12. Li, J.-Q. Battery-electric transit bus developments and operations: A review. Int. J. Sustain. Transp. 2014, 10, 157-169. [CrossRef]

13. Kim, E.; Lee, J.; Shin, K.G. Real-time prediction of battery power requirements for electric vehicles. In Proceedings of the ACM/IEEE 4th International Conference on Cyber-Physical Systems-ICCPS '13, Philadelphia, PA, USA, 8-11 April 2013; Association for Computing Machinery (ACM): New York, NY, USA, 2013; pp. 11-20.

14. Montgomery, J.; Kusano, K.D.; Gabler, H.C. Age and gender differences in time to collision at braking from the 100-car naturalistic driving study. Traffic Inj. Prev. 2014, 15, S15-S20. [CrossRef] [PubMed] 
15. Dingus, T.A.; Guo, F.; Lee, S.; Antin, J.F.; Perez, M.; Buchanan-King, M.; Hankey, J. Driver crash risk factors and prevalence evaluation using naturalistic driving data. Proc. Natl. Acad. Sci. USA 2016, 113, 2636-2641. [CrossRef] [PubMed]

16. Hamzeie, R.; Savolainen, P.T.; Gates, T.J. Driver speed selection and crash risk: Insights from the naturalistic driving study. J. Saf. Res. 2017, 63, 187-194. [CrossRef] [PubMed]

17. Wang, S. Evaluation of Traffic Speed Control Devices and Its Applications. Ph.D. Dissertation, Iowa State University, Ames, IA, USA, 2018.

18. Zoepf, S.; MacKenzie, D.; Keith, D.; Chernicoff, W. Charging choices and fuel displacement in a large-scale demonstration of plug-in hybrid electric vehicles. Transp. Res. Rec. J. Transp. Res. Board 2013, 2385, 1-10. [CrossRef]

19. Jabeen, F.; Olaru, D.; Smith, B.; Braunl, T.; Speidel, S. Electric vehicle battery charging behaviour: Findings from a driver survey. In Proceedings of the 36th ATRF Australian Transportation Research Forum, Brisbane, Australia, 2-4 October 2013.

20. Sun, X.-H.; Yamamoto, T.; Morikawa, T. Charge timing choice behavior of battery electric vehicle users. Transp. Res. Part D Transp. Environ. 2015, 37, 97-107. [CrossRef]

21. Liu, K.; Wang, J.; Yamamoto, T.; Morikawa, T. Modelling the multilevel structure and mixed effects of the factors influencing the energy consumption of electric vehicles. Appl. Energy 2016, 183, 1351-1360. [CrossRef]

22. Doshi-Velez, F.; Kim, B. Towards a rigorous science of interpretable machine learning. arXiv 2017, arXiv:1702.08608.

23. She, C.; Wang, Z.; Sun, F.; Liu, P.; Zhang, L. Battery aging assessment for real-world electric buses based on incremental capacity analysis and radial basis function neural network. IEEE Trans. Ind. Inform. 2020, 16, 3345-3354. [CrossRef]

24. Xydas, S.; Hassan, A.; Marmaras, C.; Jenkins, N.; Cipcigan, L. Electric vehicle load forecasting using data mining methods. In Proceedings of the Hybrid and Electric Vehicles Conference 2013 (HEVC 2013), London, UK, 6-7 November 2013; pp. 10-11. [CrossRef]

25. Majidpour, M.; Qiu, C.; Chu, P.; Gadh, R.; Pota, H.R. A novel forecasting algorithm for electric vehicle charging stations. In Proceedings of the 2014 International Conference on Connected Vehicles and Expo (ICCVE), Vienna, Austria, 3-7 November 2014; Institute of Electrical and Electronics Engineers (IEEE): Piscataway Township, NJ, USA, 2014; pp. 1035-1040.

26. Li, Y.; Zou, C.; Berecibar, M.; Nanini-Maury, E.; Chan, J.C.-W.; Bossche, P.V.D.; Van Mierlo, J.; Omar, N. Random forest regression for online capacity estimation of lithium-ion batteries. Appl. Energy 2018, 232, 197-210. [CrossRef]

27. Li, C.; Chen, Z.; Cui, J.; Wang, Y.; Zou, F. The lithium-ion battery state-of-charge estimation using random forest regression. In Proceedings of the 2014 Prognostics and System Health Management Conference (PHM-2014 Hunan), Zhangjiajie, China, 24-27 August 2014; pp. 336-339. [CrossRef]

28. Zheng, Z.; Peng, J.; Zhang, X.; Deng, K.; Gao, K.; Li, H.; Chen, B.; Yang, Y.; Huang, Z. A novel method for lithium-ion battery remaining useful life prediction using time window and gradient boosting decision trees. In Proceedings of the ICPE 2019-ECCE Asia and 10th International Conference on Power Electronics, Bexco, Busan, Korea, 27-30 May 2019; Volume 3, pp. 3297-3302.

29. Jiang, F.; Yang, J.; Cheng, Y.; Zhang, X.; Yang, Y.; Gao, K.; Peng, J.; Huang, Z. An Aging-Aware SOC Estimation Method for Lithium-Ion Batteries using XGBoost Algorithm. In Proceedings of the 2019 IEEE International Conference on Prognostics and Health Management (ICPHM), San Francisco, CA, USA, 17-20 June 2019; Institute of Electrical and Electronics Engineers (IEEE): Piscataway Township, NJ, USA, 2019; pp. 1-8.

30. Ma, F.; Yan, X. Research on the Energy Consumption Estimation Method of Pure Electric Vehicle Based on XGBoost. In Proceedings of the 2019 3rd International Conference on Electronic Information Technology and Computer Engineering (EITCE), Beijing, China, 18-20 October 2019; Institute of Electrical and Electronics Engineers (IEEE): Piscataway Township, NJ, USA, 2019; pp. 1021-1026.

31. UCLA; Statistical Consulting Group. Introduction to Generalized Linear Mixed Models. 2018. Available online: https://stats.idre.ucla.edu/other/mult-pkg/introduction-to-generalized-linear-mixed-models/ (accessed on 28 November 2020).

32. Bates, D.; Mächler, M.; Bolker, B.; Walker, S. Fitting linear mixed-effects models using lme4. J. Stat. Softw. 2015, 67, 1-48. [CrossRef] 
33. Storn, R.; Price, K. Differential evolution-A simple and efficient heuristic for global optimization over continuous spaces. J. Glob. Optim. 1997, 11, 341-359. [CrossRef]

34. Zhu, H. Numerical study of urban traffic flow with dedicated bus lane and intermittent bus lane. Phys. A Stat. Mech. Its Appl. 2010, 389, 3134-3139. [CrossRef]

35. Surprenant-Legault, J.; Elgeneidy, A.M. Introduction of reserved bus lane. Transp. Res. Rec. J. Transp. Res. Board 2011, 2218, 10-18. [CrossRef]

Publisher's Note: MDPI stays neutral with regard to jurisdictional claims in published maps and institutional affiliations.

(C) 2020 by the authors. Licensee MDPI, Basel, Switzerland. This article is an open access article distributed under the terms and conditions of the Creative Commons Attribution (CC BY) license (http://creativecommons.org/licenses/by/4.0/). 